This is a post-peer-review, pre-copyedit version of a paper published in New Trends in Educational Activity in the Field of Mechanism and Machine Theory, MMS 64 : 64-71 (2019). The final authenticated version is available online at: http://dx.doi.org/10.1007/978-3-030-00108-7 8 .

\title{
Dynamics and Mechanical Vibrations. Complementing the Theory with Virtual Simulation and Experimental Analysis
}

\author{
M. Urízar ${ }^{1}$, V. Petuya ${ }^{1}$, A. Hernández ${ }^{1}$ and E. Macho ${ }^{1}$
}

${ }^{1}$ Faculty of Engineering in Bilbao, Dpt. of Mechanical Engineering, University of the Basque Country (UPV/EHU), Spain, e-mail: monica.urizar@ehu.es

\begin{abstract}
The purpose of the present work is to show how the complex theory in subjects related to Mechanism and Machine Theory can be reinforced with some practicals in which the students can perform virtual simulation of the mechanisms under study, and they can even interact with real prototypes to validate by means of experimental analysis the theoretical results. This works deals with Dynamics and Mechanical Vibrations, and presents the capacities of the Dynamics module implemented in GIM software related to the obtaining of free solid diagrams, internal forces maps and diagrams, motion simulation, and so on. In addition to this, interacting with prototypes to carry out experimental measures is also proposed, so that the students can acquire a deeper understanding of some phenomena related to mechanical vibrations.
\end{abstract}

Key words: Education, Dynamics, Mechanical vibrations, Virtual simulation, Experimental analysis

\section{Introduction}

Basing on our own experience [1], specific computer programs constitute a very helpful tool to help students to easily achieve a better comprehension of the theoretical concepts explained in the lectures [2,3]. Indeed, currently, most of the textbooks related to Mechanism and Machine Theory include simulation programs to reinforce and complement the contents of the book [4,5]. 
The subject this work deals with, Mechanism Theory and Mechanical Vibrations, plays an essential role in education on Mechanical Engineering. In the Faculty of Engineering in Bilbao, University of the Basque Country (UPV/EHU), this subject is taught during the third course in Mechanical Engineering [6,7], and it covers significant topics, such as, planar motion geometry, analytic methods, dimensional synthesis, dynamics (inverse and direct dynamics), flywheels, mechanical vibrations, and so on. In general, students encounter some difficulties when facing up to the complex concepts intrinsic to the theoretical bases.

We presented in [1] a work in which virtual simulation in GIM software [3] enabled the students to go deeper into the kinematics of planar mechanisms. Following with this effort, we propose now two main actions to facilitate the understanding of the second part of the subject. On the one hand, use GIM software to perform virtual simulation of the Dynamics problem, and, on the other hand, complement the theory of mechanical vibrations with experimental analysis with real prototypes. GIM is a registered software created by the COMPMECH Research Group belonging to the Department of Mechanical Engineering of UPV/EHU. The software is intended for educational purposes. Until now the modules implemented in the software dealt with kinematic analysis, motion simulation and synthesis. Currently, another module, called Dynamics, has been incorporated [8] and it is precisely this module the one we propose to reinforce the part of the subject in which the dynamic problem is studied.

Regarding the mechanical vibrations, students carry out some practicals in which they can interact with real prototypes and they can visualize the vibration modes of certain mechanical systems, they can assess the resonance phenomena, they can understand the damping effect, and so on. The main target is to acquire a complete vision of the subject, from theory to practice, which, in our opinion, is the correct way to really understand the subject.

\section{Dynamics. Virtual simulation in GIM software}

Bearing in mind the objective of helping the students to better understand the concepts illustrated in the subject, the theoretical lectures are complemented and reinforced with practical exercises. On the one hand, in the laboratory, virtual simulation of the dynamics behaviour of certain mechanisms is carried out in GIM software. On the other hand, the students, at the time of studying the subject on their own, can freely download the software from the web site of COMPMECH Research Group: http://www.ehu.eus/compmech/software/. In this way, they can check and validate the results they theoretically obtain when solving the dynamics problem. 


\subsection{Capacities of Dynamics module}

A screenshot of the main window of Dynamics module in GIM is shown in Fig. 1. All the modules implemented in GIM offer an easy-to-use format in which the user can navigate through all the existing options and simply select the ones he/she is interested in.

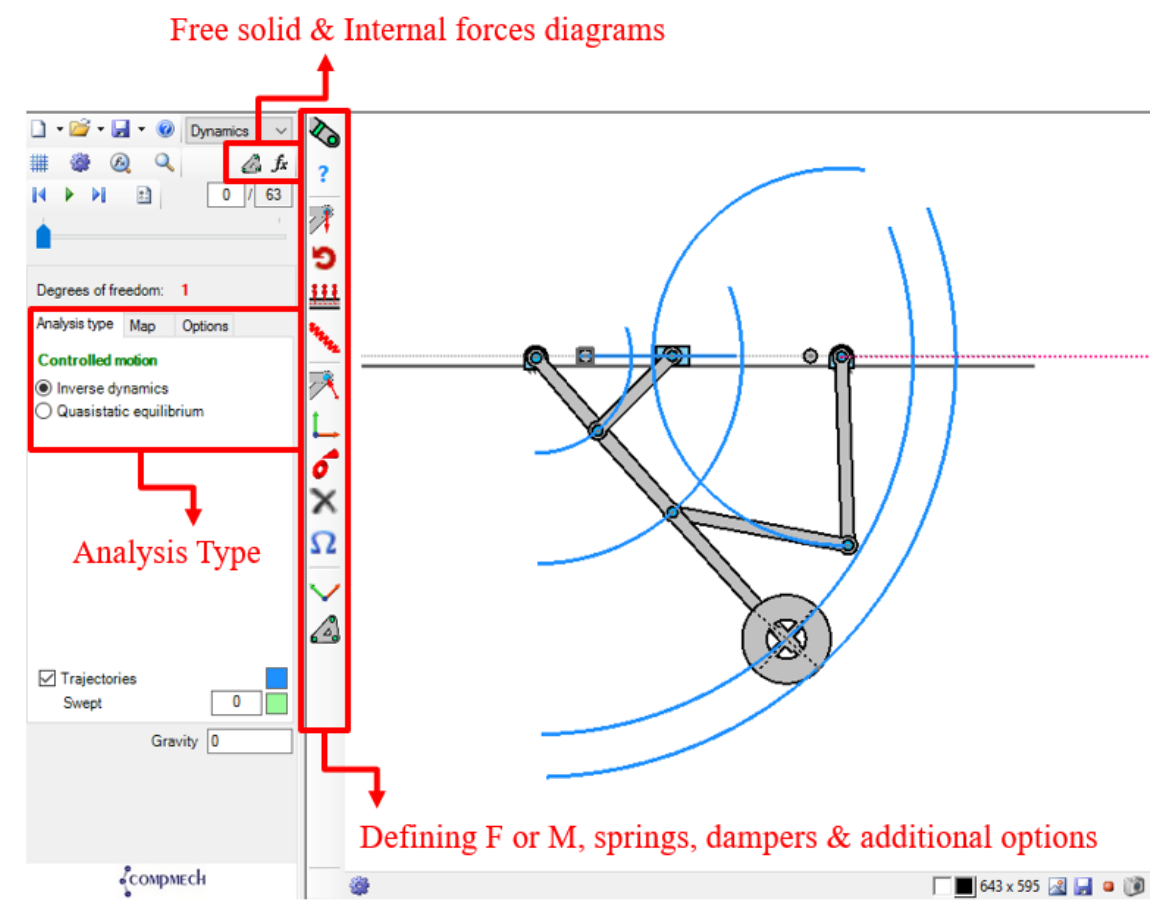

Fig. 1 Main window of Dynamics module in GIM software

As an illustrative example, a landing gear mechanism of an aircraft has been modelized (see Fig. 1) and the trajectories of points of interest can be visualized. For this first step, the geometry of the mechanism has been already created and its motion has been simulated. Both actions are achieved by using the modules called $\mathrm{Ge}$ ometry and Kinematics. Students are already familiarized with these modules as they are used in the first part of the subject which deals with kinematics [1].

One of the analysis types that the Dynamics module includes is the Inverse Dynamics. It enables the user to obtain the necessary forces or moments to actuate the 
mechanism once the motion is known and the set of applied loads have been predefined. These applied loads can be punctual forces or moments, as well as linearly distributed forces. As the motion has been already obtained, the acceleration of the gravity centre and the angular acceleration of each moving element is known in all the poses of the mechanism. Then, once the mass properties are defined, the inertial loads are computed.

Basing on D'Alembert principle, in which the inertial loads are treated as external virtual loads, the free solid diagrams of any element of the mechanism can be obtained. As an example, Fig. 2 shows the free solid diagram of one of the bars of the landing gear mechanism. It is important to emphasize that these diagrams can be obtained for any pose of the mechanism. In this way, the students can visualize the evolution of the diagrams along the motions of the mechanism. Another analysis type implemented in the module is the one called Quasi-static equilibrium. It works in a very similar way to the Inverse Dynamics with the difference that in the quasistatic one the inertial loads are considered null.

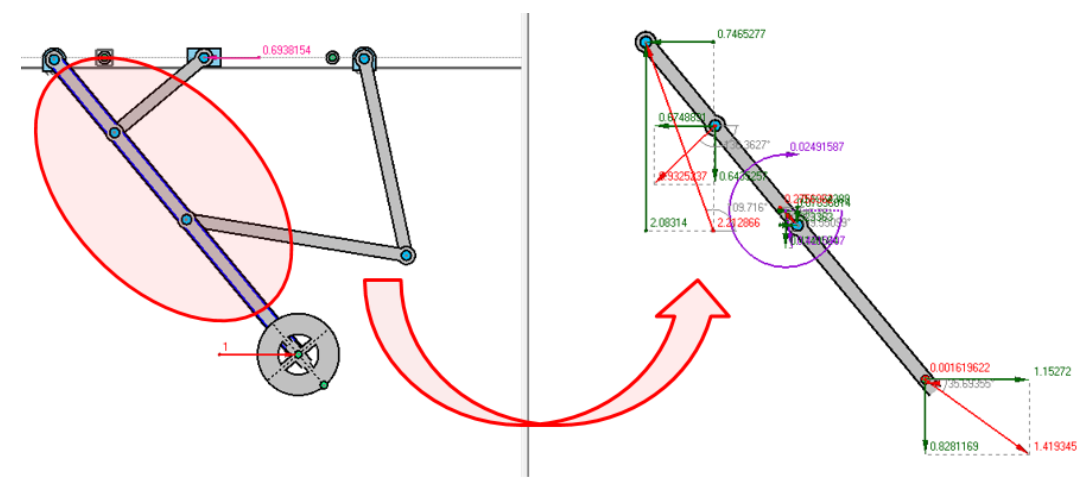

Fig. 2 Free solid diagrams

Additionally, the internal forces of any element of the mechanism can be computed. Two main options are offered to the user. The first one is to visualize the maps of the internal forces of all the elements of the mechanism. This is shown in Fig. 3a, in which coloured maps associated to the axial forces, the shear forces and the bending moments are plotted. The second option is to represent the diagrams of the internal forces for a selected element, as it is depicted in Fig. 3b.

Dynamics module also implements another analysis type called Direct dynamics. The target of this approach is to obtain the motion of the mechanism once the set of actuating and applied loads is predefined and some initial conditions are established. In this case, the user only defines the geometry of the mechanism under study (in Geometry module) and then, once inside Dynamics module, selects the option Direct 
dynamics. The user then defines the set of loads applied to the mechanism, the initial conditions of position and velocity, and the software automatically computes the corresponding motion. An illustrative example is shown in Fig. 4, where the motion of a bar connected by springs to the fixed element and subjected to its own weight can be visualized.

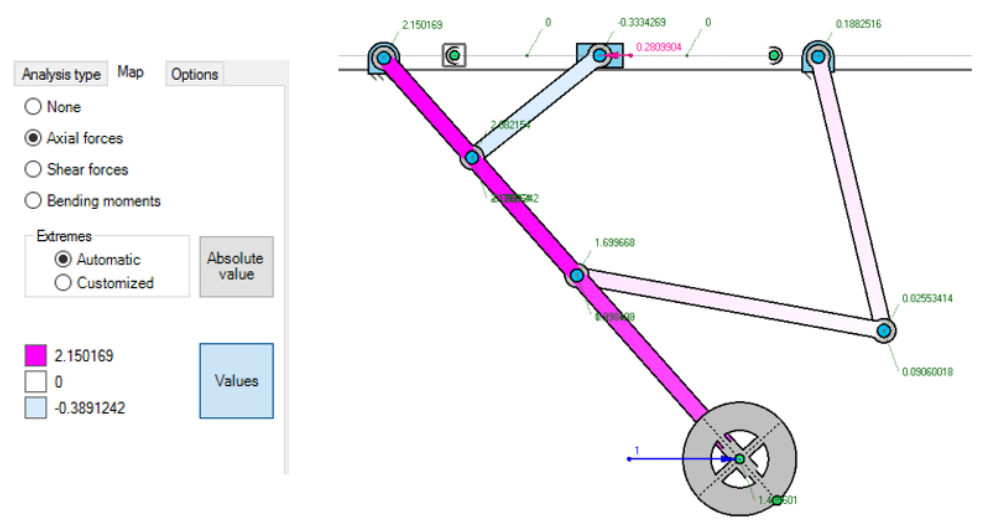

a)
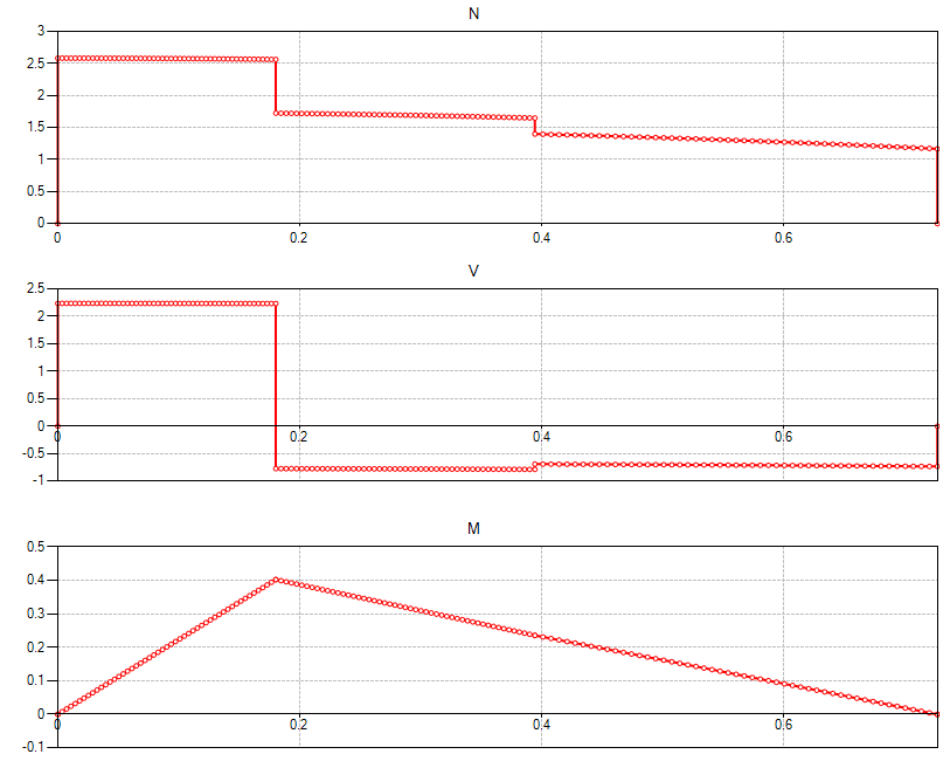

b)

Fig. 3 Internal forces maps and diagrams 


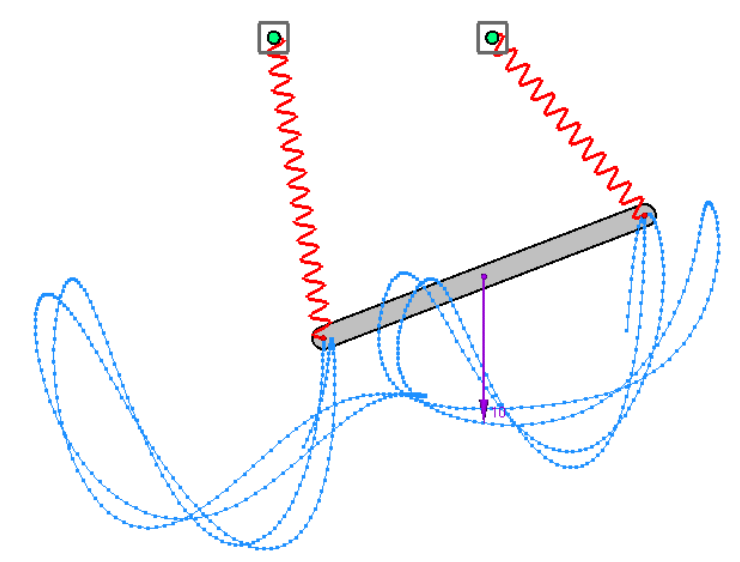

Fig. 4 Motion simulation in Direct dynamics

\section{Vibration experimental analysis interacting with prototypes}

The second part of the subject Mechanism Theory and Mechanical Vibrations deals with the phenomena of free or forced mechanical vibrations of single and multiple degree-of-freedom mechanical systems. In relation to forced mechanical vibrations, the response of the system to harmonic type excitations, as well as the response to step, pulse and transient vibration excitations and their combinations is studied. Modal analysis technique is also studied for multiple dof mechanical systems.

Apart from this lengthy theory, some concepts regarding the experimental analysis of mechanical vibrations are explained during the lectures. Additionally, the theoretical concepts are reinforced with some practicals in the lab, in which the students interact with real prototypes and carry out experimental measures. To cite some examples, the students visualize the vibration modes of a beam making use of a stroboscope, they obtain the natural frequencies of a prototype of a building using an impact hammer and analyzing the response measured by the accelerometers, they assess the vibration modes of the prototype being subjected to a harmonic force varying its frequency and they analyse the resonance phenomenon of a single dof mechanical system.

One of these aforementioned practices is shown in Fig. 5. In this Fig. 5a, the prototype of a single dof mechanical system actuated by a slider-crank mechanism driven by an electric motor is displayed. In this case the vibrations are induced to the mass due to the motion imposed on the slider. The students obtain experimentally the 
displacement of the mass as a function of the frequency of the input (the angular velocity of the crank) and confirm the high amplitude the mass acquires when getting close to the natural frequency (see Fig. 6). These results are validated with the ones they obtain by developing the theoretical equations, and they are also validated with the simulation of the mechanical system using GIM (Fig. 5b).

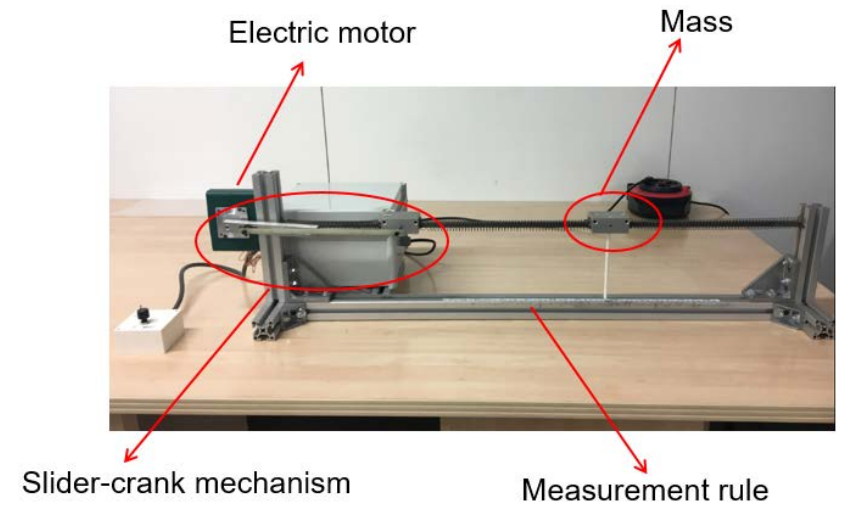

a)

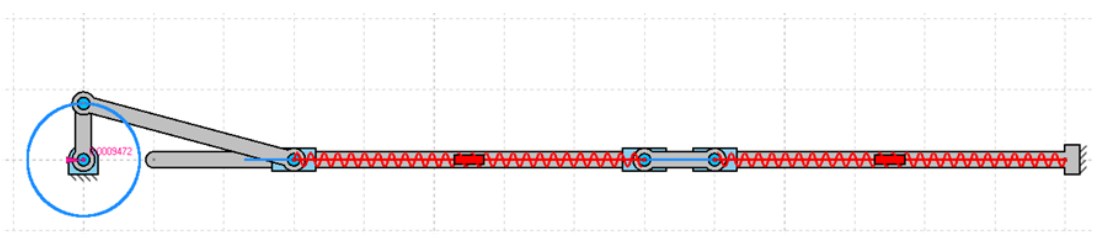

b)

Fig. 5 Mechanical vibrations: a) Prototype and b) simulation
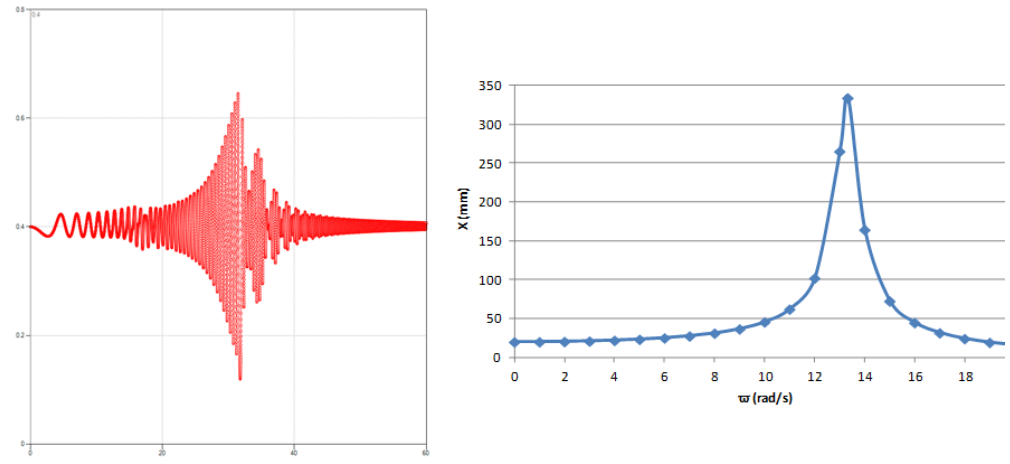

Fig. 6 Validating experimental results 


\section{Conclusions}

The work presented in this paper is a summary of some of the actions proposed by the teaching staff of the subject Mechanism Theory and Mechanical Vibrations with the purpose of facilitating the students a deeper comprehension of relevant concepts, in particular, the ones related to Dynamics and Mechanical Vibrations. On the one hand, the Dynamics module implemented in GIM software enables the students to perform simulations of many different mechanisms, obtaining the free solid diagrams and maps, the values of the actuating loads, the motion of the mechanism under certain loads. On the other hand, mechanical vibrations theory is complemented with experimental analysis so as to understand the insights of important concepts, such as resonance, damping effects, vibration modes, and so on. Moreover, the combination of simulation in GIM and experimental analysis becomes a strong tool for the students when facing up to the subject.

Acknowledgments This work was supported by the Spanish Government through the Ministerio de Economía y Competitividad (Project DPI2015-67626-P (MINECO/FEDER, UE)), the financial support from the University of the Basque Country (UPV/EHU) under the program UFI 11/29 and the support to the research group, through the project with ref. IT949-16, given by the Departamento de Educación, Política Lingüística y Cultura of the Regional Government of the Basque Country.

\section{References}

1. Urízar, M., Altuzarra, O., Diez, M., Campa, F. and Macho, E.: New Trends in Educational Activity in the Field of Mechanism and Machine Theory. Chapter: Kinematic Analysis of Planar Mechanisms by Means of Examples. Springer (J.C. García Prada, C. Castejón), 2014.

2. Pennestrì, E. and Vita, L.: Advances in Computational Multibody Systems, Chapter: Multibody dynamics in advanced education, pp. 345-370. Springer Netherlands, 2005.

3. Petuya, V., Macho, E., Altuzarra, O., Pinto, Ch. and Hernández, A.: Educational Software Tools for the Kinematic Analysis of Mechanisms - Computer Applications in Engineering Education, doi: 10.1002/cae.20532, 2011.

4. Waldron, K.J. and Kinzel, G. L.: Kinematics, dynamics and design of machinery. John Wiley \& Sons, Inc, New York, 1999.

5. Norton, R. L.: Design of machinery: An introduction to the synthesis and analysis of mechanisms and machines. McGraw-Hill, Boston, 2003.

6. Hernández, A.: Cinemática de mecanismos: Análisis y Diseño, Editorial Síntesis, Madrid, 2010.

7. Hernández, A., Pinto, Ch., Agirrebeitia, J. and Petuya, V.: Dinámica de máquinas. Publicaciones dela Escuela de Ingeniería de Bilbao, 2013.

8. Macho, E., Petuya, V., Urízar, M. and Hernández, A.: Software de propósito general para resolver la dinámica inversa y calcular los esfuerzos internos de mecanismos planos, $13^{\circ}$ Congreso Iberoamericano de Ingeniería Mecánica, CIBEM2017, Lisboa, Portugal, 2017. 